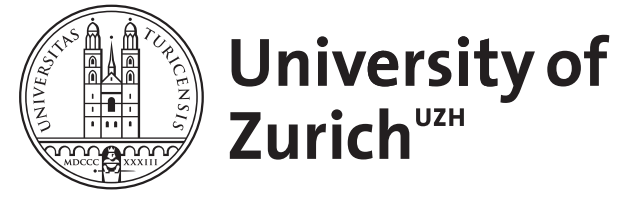

Zurich Open Repository and Archive

University of Zurich

University Library

Strickhofstrasse 39

CH-8057 Zurich

www.zora.uzh.ch

Year: 2015

\title{
La philologie linguistique et éditoriale
}

Glessgen, Martin-Dietrich

Posted at the Zurich Open Repository and Archive, University of Zurich ZORA URL: https://doi.org/10.5167/uzh-134210

Book Section

Published Version

Originally published at:

Glessgen, Martin-Dietrich (2015). La philologie linguistique et éditoriale. In: Schweickard, Wolfgang; PolzinHaumann, Claudia. Manuals of Romance Linguistics. Berlin/Boston: de Gruyter, 108-130. 


\section{Manuals of Romance Linguistics}

Manuels de linguistique romane Manuali di linguistica romanza Manuales de lingưística románica

Edited by

Günter Holtus and Fernando Sánchez Miret

\section{Volume 8}




\section{Manuel de linguistique française}

Édité par

Claudia Polzin-Haumann et Wolfgang Schweickard 
ISBN 978-3-11-030208-0

e-ISBN (PDF) 978-3-11-030221-9

e-ISBN (EPUB) 978-3-11-039413-9

\section{Library of Congress Cataloging-in-Publication Data}

A CIP catalog record for this book has been applied for at the Library of Congress.

\section{Bibliographic information published by the Deutsche Nationalbibliothek}

The Deutsche Nationalbibliothek lists this publication in the Deutsche Nationalbibliografie; detailed bibliographic data are available on the Internet at http://dnb.dnb.de.

(C) 2015 Walter de Gruyter GmbH, Berlin/Boston

Cover image : ( ) Marco2811/fotolia

Typesetting : jürgen ullrich typosatz, Nördlingen

Printing and binding : $\mathrm{CPI}$ books $\mathrm{GmbH}$, Leck

@ $\otimes$ Printed on acid-free paper

Printed in Germany

www.degruyter.com 



\title{
Hélène Carles et Martin Glessgen
}

\section{La philologie linguistique et éditoriale}

\begin{abstract}
La philologie comporte la critique textuelle ainsi que les analyses littéraire et linguistique des textes édités. Les objectifs et méthodologies de ces trois aspects de la philologie sont distincts. Le présent article met en relief le rôle de la «philologie linguistique » dans l'établissement du texte et dans sa description. Le traitement linguistique des textes oïliques médiévaux implique les paramètres de l'espace, du temps et des genres textuels (textes littéraires religieux et profanes, textes d'un savoir spécialisé, textes documentaires) ainsi que les différents aléas de la production textuelle. L'établissement des textes peut faire appel à différents types d'édition, mais repose toujours sur une analyse des divers domaines du langage (grapho-phonétique, morphologie, lexique, éventuellement syntaxe textuelle). La philologie linguistique dispose d'une méthodologie établie et efficace, mais souffre d'une faible présence dans l'enseignement universitaire.
\end{abstract}

Keywords : philologie, ecdotique, genres textuels, lexicographie historique, variation diasystématique

\section{Définition de la philologie (linguistique)}

Le terme de philologie comprend au sens large, et initialement, l'intégralité des sciences littéraires et linguistiques. C'est le sens des concepts allemand Romanische Philologie et italien Filologia romanza. Dans le sens étroit, actuellement plus répan$\mathrm{du}$, il se réfère à la théorie et la pratique éditoriales qui comportent la critique textuelle, mais également des pans interprétatifs. Il existe une distinction de fait entre une philologie "littéraire» (plus intéressée par les aspects de construction littéraire, de mise en forme textuelle, de stylistique ou de métrique) et une philologie «linguistique» (plus ciblée sur la description des systèmes grapho-phonétiques, morphologiques et syntaxiques et sur les aspects lexicaux). Il est certain que la pratique éditoriale gagne à ne pas opérer de frontière étanche entre ces deux philologies, mais il est aujourd'hui patent que l'on a affaire à deux univers disciplinaires et méthodologiques distincts (cf. Wilhelm 2015, §1 qui décrit en détail les différents aspects de ce clivage).

La philologie linguistique est, certes, pratiquée, mais elle n'a jamais été érigée en système (citons p. ex. les travaux de F. Zufferey ou d'A. Varvaro ou encore les réflexions de G. Contini qui sont à la base du «néo-lachmannisme » italien ; cf. Contini 2007, notamment 75-97, La critica testuale come studio di strutture [1971]). Or, c'est seulement une fois admise la scission entre philologie littéraire et linguistique qu'il devient possible de définir le rôle de la philologie linguistique autant dans l'établissement et la compréhension du texte que dans la définition de son ancrage spatio- 
temporel. La philologie littéraire a trop longtemps négligé les implications linguistiques ne serait-ce que du travail strictement éditorial. Les scientifiques littéraires ont une telle familiarité des textes qu'ils en ont une connaissance presque intuitive. On pourrait presque dire que, par tradition, ils ne perçoivent pas l'altérité du savoir linguistique puisque la compréhension des textes médiévaux est dans leur discipline un préalable sine qua non. Mettre en relief l'importance de la linguistique pour la philologie éditoriale et préciser les interactions entre l'analyse linguistique et l'établissement des textes, contient pourtant un potentiel notable pour la philologie des prochaines années.

Indépendamment de ses orientations plus spécifiques, la philologie éditoriale est une science moins doctrinale que pratique : elle s'exprime par la publication d'éditions de textes et par la réflexion sur les problèmes qui lui sont inhérents bien plus que par une réflexion abstraite et théorique. La complexité de la philologie s'explique par la multitude des cas de figure concrets, par la diversité des disciplines impliquées (littérature, linguistique, histoire) et par les différentes finalités de chacune d'entre elles. S’ajoute par ailleurs sa dimension internationale : la philologie dont la langue d'objet est le français est exercée notamment, en dehors des pays francophones, en Italie, en Allemagne, aux Pays-Bas, dans les pays scandinaves et anglo-américains (cf. Duval 2006). Les nombreuses traditions nationales suivent partiellement des voies différentes, générant ainsi une science «pluricentrique ». L'absence de méthodologie commune a été souvent reprochée à la philologie, mais cette absence est intrinsèque à la discipline ; la philologie consiste plus en un faisceau de règles méthodologiques qu'en une doctrine homogène.

La philologie éditoriale couvre naturellement toute la trajectoire diachronique $\mathrm{du}$ français, du IX ${ }^{\mathrm{e}}$ siècle jusqu'aujourd'hui. Elle a toutefois connu un développement particulier pour le français médiéval en se concentrant par tradition non seulement sur les textes pleins (cf. infra, 3.2), mais encore sur les textes littéraires. L'extension et la richesse de la production écrite du français médiéval ne sauraient pourtant être réduites à la seule expression littéraire. Dès le Moyen Âge, la complexité des traditions textuelles s'exprime avec une grande intensité (cf. infra, 3.3). Cette période a par conséquent suscité les interrogations philologiques les plus épineuses, même si la Renaissance, les XVII ${ }^{\mathrm{e}}$ et XVIII ${ }^{\mathrm{e}}$ ou les $\mathrm{XIX}^{\mathrm{e}}$ et $\mathrm{XX}^{\mathrm{e}}$ siècles demeurent des champs d'études passionnants.

\section{Orientation bibliographique}

La diversité interne de la philologie explique l'immensité de la bibliographie ayant pour objet cette discipline. Ce constat vaut déjà pour le seul domaine de la langue d'oïl médiévale traité ici. Les répertoires disponibles sont assez complets pour la littérature profane; concernant les autres genres, ils fournissent des informations plus partielles : 
- $\quad$ le Grundriß der romanischen Literaturen des Mittelalters (GRLMA 1968-) donne le meilleur aperçu interprétatif pour les différents genres textuels de la littérature profane ainsi que pour les sources historiographiques (vol. 11/1-11/3);

- $\quad$ le Manuel bibliographique de la littérature française du Moyen Âge de R. Bossuat et de ses successeurs (1951-1986) est presque exhaustif jusqu'au milieu des années 1980; il est complété ensuite par la Bibliographie der französischen Literaturwissenschaft d'O. Klapp et de sa fille A. Klapp-Lehmann (1960-) ;

- les bibliographies des grands dictionnaires médiévaux intègrent également dans une mesure significative la littérature religieuse et celle d'un savoir spécialisé (cf. infra, 3.4) ;

- d'autres genres très spécifiques ont été traités par la Typologie des sources du Moyen Âge occidental de L. Génicot (TypSources, 1972-) ;

- l'Inventaire systématique (InvSyst, 1997) décrit la quasi-totalité des manuscrits connus antérieurs à 1250, tous genres textuels confondus (complétés, pour les textes documentaires, par la collection des DocLing, 1204-ca 1300);

- $\quad$ enfin, l'Institut de recherche et d'histoire des textes (IRHT) réunit un nombre considérable d'informations sur des manuscrits individuels et ses fichiers sont en voie de numérisation (cf. <irht.cnrs.fr>).

Les ouvrages de type interprétatif sont légion et ne pourront être mentionnés ici que de manière très ponctuelle. On se reportera notamment, pour de plus amples renvois bibliographiques, au manuel dédié intégralement à la Philologie de l'édition dans la collection des Manuals of Romance Linguistics (MRL 4, ed. D. Trotter), auquel nous renvoyons de manière systématique. De nombreux aspects de linguistique diachronique, sous-jacents à la philologie linguistique, ont été traités par ailleurs dans la Romanische Sprachgeschichte (RSG, 2003-2008, cf. art. 7, 103, 107, 116, 121, 128, 175, 181, 185, 187, 192, 197, 198).

\section{Les objets d'observation}

\subsection{L’espace du français écrit médiéval}

Le français médiéval est mis à l'écrit au Bas Moyen Âge dans pratiquement toutes les régions où il est également parlé. L'espace dialectal oïlique tel qu'il se présente au début du $\mathrm{XX}^{\mathrm{e}}$ siècle dans l'ALF permet une première approche de cette distribution. La tradition médiéviste distingue dans cet espace deux ensembles «scriptologiques », l'un plutôt occidental, l'autre plutôt oriental : les régions plutôt «occidentales » concernent la Normandie, Paris et l'Île-de-France, l'Ouest et la Bretagne romane, le Centre (comprenant l'Anjou, la Touraine et l'Orléanais) et enfin le Sud-Ouest (Vendée, Saintonge et Poitou). Les régions plutôt « (nord-)orientales » sont le Nord et la Flandre 
française, la Picardie, la Wallonie, la Champagne, la Lorraine, la Franche-Comté, la Bourgogne ainsi que le Bourbonnais (cf. Pfister 1993).

Les analyses dialectométriques récentes à partir des deux atlas de Dees (1980 ; 1987) montrent toutefois un paysage scriptologique plus complexe (cf. Goebl 2012, cartes 17s., 23s.) et plus en cohérence avec la nature linguistique des textes oïliques. Les textes médiévaux ne reflètent pas les variétés dialectales de l'oral, mais correspondent à des variétés régionales semi-artificielles. Celles-ci s'élaborent jusqu'au XIVe siècle dans la logique d'une codification pluricentrique avant de connaître une homogénéisation plus importante, entre autres sous l'impact du modèle parisien (cf. Glessgen 2012b ; ત1 Le français dans l'histoire : depuis ses origines jusqu'au XVI' siècle).

Quant à l'espace géolinguistique concerné, deux remarques complémentaires s’imposent :

- d'une part, le Poitou n'a pas appartenu tout au long de son histoire à l'espace linguistique oïlique. Initialement occitans, les parlers poitevins se sont orientés de manière significative vers le français, après l'an 1000 ou 1100 (Pignon 1960, 516);

- le cas de Paris et de l'île-de-France (qui n'est plus dialectophone depuis au moins le XVIII ${ }^{e}$ siècle) soulève d'autres problèmes : cet ensemble est souvent terminologisé comme « francien », mais il n'existe pratiquement pas de texte vernaculaire de Paris avant le milieu $\mathrm{du}$ XIII ${ }^{\mathrm{e}}$ siècle. Cela s'explique notamment par le fait que la mégapole réunissait un grand nombre de personnes formées qui ont longtemps fait appel exclusivement à la langue de prestige qu'était le latin (cf. Videsott 2013 ; Grübl 2013). Ainsi, Paris n’a pas pu jouer de rôle réel dans le paysage de l'écrit oïlique avant $c a 1300$. Le terme « francien » ne recouvre donc aucune réalité linguistique avant le XIV ${ }^{\mathrm{e}}$ siècle ; son utilisation pour l'époque postérieure reste aussi sujette à caution.

En dehors de ces régions où le français est la langue héréditaire, l'écrit oïlique est également présent, pour des raisons extralinguistiques, en territoire francoprovençal, en Angleterre, dans l'Orient latin et en Italie du Nord :

- Le français est quasiment exclusif à l'écrit dans l'intégralité de l'espace francoprovençal. Les fonctions communicatives se répartissent entre les dialectes francoprovençaux d'une part qui constituent la langue de communication quotidienne et une scripta française de type sud-oriental, qui est « importée » en raison de son prestige sociolinguistique. L'écrit oïlique a longtemps été la seule partie visible et il est difficile de définir précisément quel fut l'espace linguistique francoprovençal à l'époque médiévale. Des recherches philologiques et toponymiques récentes ont permis de préciser l'extension du francoprovençal à l'oral qui s'est avérée bien plus large qu'aujourd'hui (en partie la Franche-Comté, la Bourgogne - y inclus Cluny -, la Suisse romande ainsi que la grande région autour de Lyon ; cf. Zufferey 2006; Chambon/Müller 2013; cf. aussi, pour le domaine aujourd'hui alémanique, Kristol 2002).

- L'implantation du français en Angleterre est essentiellement liée à la conquête normande (1066). Il s'agissait alors d'une langue parlée parmi une élite militaire, politique et culturelle. Sa présence à l'oral est très minoritaire (environ 10.000 colons francophones cohabitent 
avec près de 1,5 million d'habitants autochtones), mais elle est stable jusqu'au milieu du XIII ${ }^{\mathrm{e}}$ siècle et peut-être au-delà. À l'écrit, le français occupe une place plus importante qu'à l'oral et pendant plus longtemps, l'anglais ne devenant majoritaire qu'à partir de ca 1430. La scripta française pratiquée en Angleterre est terminologisée comme anglo-normand, même si une appellation neutre comme français insulaire serait plus adéquate étant donné la diversité de la provenance régionale des immigrants francophones (cf. Trotter 2012; 2013 [2014]).

- $\quad$ En Orient latin, le français est utilisé comme langue acrolectale surtout aux XII ${ }^{\mathrm{e}}$ et XIII ${ }^{\mathrm{e}}$ siècles, notamment dans les États Croisés (Royaume de Jérusalem, Principauté d'Antioche, Comté de Tripoli, 1099-1291) et, plus longtemps, dans le Royaume de Chypre (1197-1489). Si le volume textuel produit en Orient est plus restreint qu'en Angleterre, il comporte néanmoins plusieurs centaines de textes appartenant à des genres divers (cf. les répertoires de Minervini 2010, 142-146 ; 2012, 99-104).

- $\quad$ La production textuelle oïlique de l'Italie du Nord aux XIII et XIV ${ }^{\mathrm{e}}$ siècles reste en revanche un phénomène très ponctuel et sans correspondance à l'oral. Les quelques dizaines de textes exclusivement littéraires, rédigés dans ce «français d'Italie » (mal terminologisé comme franco-italien ou franco-vénitien), correspondent à une scripta oillique donnée (souvent de type nord-oriental) qui comporte de nombreux italianismes, surtout lexicaux et grapho-phonétiques (cf. le recensement à jour de Holtus/Wunderli 2005 et la synthèse scriptologique de Capusso 2007).

\subsection{La chronologie textuelle et linguistique}

La périodisation du français médiéval répond, dans une optique philologique, essentiellement aux conditions de sa production textuelle. Dans ce sens, on retiendra trois moments-clé, communs aux différentes langues écrites de l’Europe médiévale :

(1) d'abord, l'émergence des langues vernaculaires à l'écrit, en contexte latin ou sous forme de micro-textes, à partir de $c a 800$,

(2) la production de textes vernaculaires pleins, à partir de ca 1100,

(3) enfin, la diffusion de l'impression à lettres mobiles transforme les conditions de la « manuscript culture » à partir de $c a 1500$ (cette date se place entre l'invention de Gutenberg en 1453 et la diffusion plus massive des livres imprimés à partir du milieu du XVI ${ }^{\mathrm{e}}$ siècle).

On distingue donc une époque préparatoire de l'écrit français médiéval comprise entre $c a 800$ et $c a 1100$ et une époque centrale comprise entre $c a 1100$ et ca 1500 qui s'ouvre enfin sur une époque moderne dont les constellations s'éloignent au fur et à mesure des paramètres médiévaux. 


\subsubsection{L’époque pré-textuelle}

Les premiers éléments romans font leur apparition à l'écrit aux alentours de 800. Cela vaut pour le français tout comme l'occitan, l'italien et les variétés ibéroromanes. Il s'agit de microtextes, souvent avec une forte charge pragmatique, mais il s'agit surtout d'éléments lexicaux, morphologiques voire infra-lexématiques insérés en contexte latin.

Entre les $\mathrm{VI}^{\mathrm{e}}$ et $\mathrm{VIII}{ }^{\mathrm{e}}$ siècles, période de leur émergence à l'oral, les langues romanes n'ont pas laissé que très peu de traces dans l'écrit (cf. néanmoins les légendes monétaires mérovingiennes, Chambon/Greub 2000). L'élaboration de la scripturalité dans les fragments vernaculaires est une conséquence de la Réforme carolingienne qui a entraîné une intensification de la culture de l'écrit. La restauration du latin normatif d'époque antique s'est ainsi accompagnée d'une plus forte conscience linguistique ; le fossé creusé entre le latin et les langues vernaculaires devint de plus en plus apparent (cf. le concile de Tours, 813). Pour pouvoir répondre aux besoins communicatifs d'un monde désormais romanophone, de nombreux éléments vernaculaires sont insérés avec un habillage graphématique et morphologique plus ou moins latinisé.

L’intégration des éléments romans concerne :

- des lexèmes souvent fréquents à l'oral (avec ou sans correspondant en latin écrit de l'Antiquité) qui ont une charge pragmatique particulière dans les documents en question,

- des toponymes dont l'identification dans les actes administratifs ou de la pratique juridique était indispensable et pour lesquels une latinisation aurait pu mettre en cause la bonne compréhension et la validité des actes,

- de manière plus restreinte, certaines marques morphologiques comme l'article défini et certains traits syntaxiques dans l'ordre des constituants.

Parallèlement aux éléments romans en contexte latin dont la nature et le rôle n'ont été décrits que récemment (cf. Chambon 1998 ; Carles 2011), apparaissent des microtextes, étudiés quant à eux très en détail par la philologie romane depuis près d'un siècle. Citons les Serments de Strasbourg (en contexte latin, 842 (?), rédaction éventuellement plus tardive, ms. fin $\mathrm{X}^{\mathrm{e}} \mathrm{s}$.), dont la romanité est indissociable d'une forte relatinisation. Ces prémices du français à l'écrit ont souvent trompé le regard des chercheurs, qui ont pu y voir l'expression d'un protofrançais alors que l'on sait les langues romanes déjà pleinement formées à cette époque. La compréhension du vernaculaire fragmentaire doit être replacée dans un contexte de l'écrit où le latin est omniprésent et jouit d'un prestige très élevé. L'émergence du français à la scripturalité ne pouvait pas ne pas en tenir compte. Les mécanismes de latinisation sont la réponse la plus habile des scribes à l'insertion progressive de la langue véhiculaire de l'oral. Un peu plus tard, la Séquence de Sainte Eulalie (texte et ms. fin du IX ${ }^{\mathrm{e}}$ siècle) témoigne d'une plus grande autonomie du français à l'écrit. Mais les témoignages textuels oíliques 
restent très rares jusqu'au $\mathrm{XI}^{\mathrm{e}}$ siècle (cf. infra, 3.3.1/3.3.2 et InvSyst 1, 311, Table chronologique).

L'émergence des langues romanes à l'époque pré-textuelle concerne presque exclusivement les genres textuels documentaires et, ponctuellement, la littérature religieuse. Par ailleurs, la production littéraire et scientifique reste attachée à la langue classique (cf. Stotz 1996-2004).

L'époque pré-textuelle est d'une importance capitale pour appréhender la nature des langues médiévales dans les premiers siècles de leur histoire et pour cerner la lente élaboration de leur système graphématique. Cette période, longtemps mise à l'écart par la linguistique historique, jette une lumière utile sur l'émergence que l'on a longtemps cru soudaine des textes littéraires romans pleins, comme la Chanson de Roland vers 1100 .

\subsubsection{La langue médiévale à l'époque textuelle}

L'époque textuelle est traditionnellement la seule considérée par la philologie car les scientifiques ont trop longtemps confondu la genèse d'une langue à l'écrit avec sa genèse à l'oral. L'époque des textes pleins s'ouvre d'emblée sur une riche production littéraire représentée entre autres par les romans en vers de Wace ou de Chrétien de Troyes. La diffusion du papier au détriment du parchemin comme support de l'écrit à partir des XIII e et XIVe siècles s'accompagne ensuite d'une forte intensification de l'écrit pour tous les genres textuels (cf. infra, 3.3). La prolifération des copies de textes à travers les siècles constitue d'ailleurs l'une des plus grandes difficultés des éditeurs modernes à cerner de manière adéquate la textualité médiévale (cf. infra, 4.1).

À l'intérieur de cette période textuelle se place, en français, le passage de l'ancien au moyen français que l'on date de manière conventionnelle aux alentours de 1350 . D'un point de vue linguistique interne, cette progression est traditionnellement définie par l'abandon de la flexion à deux cas (dont les manquements se relèvent dès les premiers témoins écrits) et par la fixation de l'ordre des constituants (SVO). La chronologie et, encore plus, la réalité ontologique de ce changement linguistique restent sujettes à caution (cf. Baum 2003). Les évolutions qui se manifestent au niveau textuel entre le XIII e et le $\mathrm{XV}^{\mathrm{e}}$ siècle sont toutefois considérables et témoignent d'une oralité vernaculaire en perpétuelle transformation.

Des problèmes de périodisation se posent également pour déterminer la fin de la période médiévale. La transformation des pratiques textuelles liées à la diffusion des livres imprimés est une donnée objective. Ce facteur externe se double toutefois de la transformation interne qu'est le passage du moyen français au français moderne. Nous ne reproduirons pas dans ce cadre toutes les étiquettes chronologiques appliquées au français (pré-classique, classique etc.) qui, dans une optique grapho-centrique, morcellent la langue sans tenir compte du caractère permanent de son évolu- 
tion. Concernant les paramètres liés au changement linguistique du français à cette époque, nous retenons comme orientation générale l'étude quantificatrice de notre regrettée amie Claire Vachon (2010) qui permet de placer entre ca 1480 et ca 1630 les transformations internes constitutives de l'élaboration de la norme classique du français.

Les questions philologiques appliquées à l'époque moderne sont bien entendu extrêmement variées, autant concernant la gestion des textes manuscrits que celle des textes imprimés (cf. p. ex. Duval 2015 pour l'époque trop négligée du XVII siècle). Enfin, la nouvelle révolution médiale de l'informatique et de l'internet, qui fait écho à la révolution de Gutenberg, clôt - toujours en termes philologiques - les cinq siècles de la période moderne comprise entre ca 1500 et ca 2000 pour s'ouvrir sur une nouvelle époque de l'écrit du français.

Ce simple aperçu met en évidence que le traitement philologique des époques pré-textuelle et textuelle fait appel à des méthodologies différentes et qu'il ouvre également d'autres perspectives, autant sur les textes que sur leurs contextes. Ici, comme ailleurs, ce sont davantage les facteurs «externes» et non de linguistique interne qui déterminent les approches philologiques.

\subsection{Les genres textuels}

L'écrit médiéval s'inscrit dans le cadre de genres textuels qui correspondent à des contextes communicatifs définis dont ils représentent une mise en forme emblématique. La structure textuelle de ces genres et, partiellement, leur vocabulaire traversent les différentes langues, et leur catégorisation pour le français est également valable pour les autres langues médiévales. De manière très générale, il est possible de distinguer cinq ensembles textuels qui sont, pour la plupart, fortement articulés (cf. pour ce chapitre GRLMA ; InvSyst ; Glessgen 2012a, 422-433).

\subsubsection{Les sources à forte charge pragmatique}

Les sources à forte charge pragmatique apparaissent comme les tout premiers témoignages vernaculaires à l'écrit. Pour le français, mentionnons le glossaire de Kassel (VIII $/ \mathrm{IX}^{\mathrm{e}} \mathrm{s}$.) et, de nouveau, les Serments de Strasbourg (842, ms. fin X $\mathrm{X}^{\mathrm{e}} \mathrm{s}$.). Ces sources restent toutefois, à l'époque pré-textuelle, très restreintes en nombre et en volume. Malgré leur transmission comme témoins uniques, ces textes ou para-textes, souvent fragmentaires et proches du latin, sont extrêmement difficiles à comprendre et à cerner d'un point de vue linguistique. Les difficultés d'interprétation et leur rôle de précurseurs expliquent que ces témoignages ont connu de nombreuses éditions et des études très minutieuses (on prendra pour exemple les 40 pages denses de Berschin/Berschin/Schmidt 1981 sur la Passion de Augsburg, longue d'une ligne de 
manuscrit, et réinterprétée par Hilty 1994 comme étant non pas du français, mais de l'occitan).

\subsubsection{La littérature religieuse}

Les textes religieux apparaissent également très tôt en français, avec des textes hagiographiques (Séquence de Sainte Eulalie fin $\mathrm{IX}^{\mathrm{e}} \mathrm{s}$. , Vie de saint Léger francooccitane $\mathrm{X}^{\mathrm{e}} \mathrm{s}$., Vie de saint Alexis fin $\mathrm{XI}^{\mathrm{e}} \mathrm{s}$., Voyage de saint Brendan déb. XII ${ }^{\mathrm{e}} \mathrm{s}$.). La littérature religieuse vernaculaire prend comme modèle le latin et connaît de nombreuses traductions, notamment de la Bible dont la transmission est souvent partielle et ne devient intégrale qu'à partir de la fin du XIII ${ }^{\mathrm{e}} \mathrm{s}$. On connaît par ailleurs des textes français liés au culte (liturgie, sermons, prières), à la spiritualité (théâtre religieux, traités moraux) ou à la doctrine (règles d'ordres religieux), alors que la discussion théologique ou la littérature pamphlétaire ne se développeront qu'à partir du XVI ${ }^{\mathrm{e}}$ siècle en langue vernaculaire (cf. pour le genre des Noëls, Rézeau 2013). Le volume textuel de la littérature religieuse est relativement important, et celle-ci a servi de modèle aux débuts de la littérature profane ; elle est toutefois bien moins étudiée à l'exception du théâtre religieux et des traductions de la Bible (cf. Buridant 2015).

\subsubsection{La littérature profane}

La littérature profane se développe en français à partir de ca 1100, avec les Chansons de gestes (Chanson de Roland, Cycle de Guillaume), suivies de près des romans en vers et des Lais, puis des romans en prose (roman arthurien), de la littérature allégorique et satirique ou des fabliaux. Les genres littéraires se diversifient notablement par la suite, surtout à partir du XIVe siècle (les genres lyriques musicaux comme les ballades, rondeaux ou complaintes, les nouvelles, les dits et débats, la littérature politique, le théâtre profane). La littérature profane française se fait forte, à travers le Bas Moyen Âge, de plusieurs milliers d'œuvres, souvent transmises par de nombreuses copies. Mais notons que c'est le seul ensemble textuel qui a connu depuis les deux derniers siècles une attention constante et intense de la part des philologues et des linguistes. Grâce aux nombreuses éditions et études, souvent très soignées, cet ensemble textuel fournit les données les plus sûres et les plus immédiatement accessibles au chercheur. Par conséquent, la littérature profane est non seulement surreprésentée en philologie éditoriale mais aussi dans les études sur l'évolution du français. 


\subsubsection{Les textes d'un savoir élaboré ou pratique}

Les textes d'un savoir élaboré réunissent des domaines de connaissances très divers, mais qui ont en commun une tradition séculaire, dans laquelle les facteurs d'imitation et de variation se répondent. La tradition la plus dense concerne les textes médicobiologiques (chirurgie, art dentaire, traités de cautérisation ; pharmacopée, herbiers ; hippiatrie et médecine des rapaces), souvent d'ascendance lointaine arabe et grecque. Les textes mathématiques et astronomiques/astrologiques sont moins nombreux, de même que les traités juridiques ou rhétoriques, plus souvent circonscrits à la langue latine. En revanche, les genres historiographiques (chroniques, annales) connaissent un certain développement en français. La plupart de ces traditions textuelles apparaissent au XII ${ }^{\mathrm{e}}$ siècle, mais se développent surtout aux XIV e $\mathrm{XV}^{\mathrm{e}}$ siècles.

Les textes d'un savoir pratique, ou - pour employer une formulation paradoxale d'un savoir "non livresque» (D. Trotter) concernent une série de petits genres de faible tradition textuelle et souvent sans antécédents latins (traités d'agriculture, de chasse, d'arpentage, de cuisine, de peinture, cartes géographiques, collections de proverbes).

Dans l'ensemble, cette multitude de textes, riche en variation lexicale plus qu'en diversité syntaxique, n'a été que partiellement prise en considération par la recherche philologique.

\subsubsection{Les textes documentaires}

L'ensemble textuel de loin le plus grand est celui des textes documentaires émanant de la gestion, de la pratique judiciaire ou de l'administration. Les premiers actes en français apparaissent vers 1200 (Douai, 1204) - après quelques rares antécédents anglo-normands (Trotter 2015, §4.3) -, mais l'utilisation de la langue vernaculaire au détriment du latin s'accélère considérablement à partir de 1300. La pratique judiciaire produit de nombreux actes (consignant surtout des ventes et des donations pieuses, des accords et des arbitrages), la gestion foncière et le commerce engendrent des relevés et listes, des livres de comptes ou encore des lettres. La diversité syntaxique et lexicale de l'écrit documentaire est considérable et largement sous-exploitée allant bien au-delà des éléments de stéréotypie, trop souvent retenus comme significatifs de ces genres.

\subsection{Les aléas de la production textuelle}

Si la chronologie des différents ensembles textuels n'est pas identique, cela vaut également pour leur distribution géolinguistique. Selon les époques et les régions, la densité et la diversité des genres peuvent varier. L'Angleterre et la Picardie, proche 
des riches villes commerçantes de la Flandre, sont précoces dans la production vernaculaire tandis que la Bourgogne avec sa forte tradition monastique ou encore Paris, centre d'érudition par excellence, restent fidèles au latin jusqu'au milieu du XIII ${ }^{\mathrm{e}}$ siècle.

Le volume global des textes peut être évalué assez précisément pour la littérature profane. Le Complément bibliographique du DEAF réunit ca 6.000 titres, la bibliographie du DMF ca 1.600, celle de l'AND ca 1.000 (chiffres qui comprennent des textes voire même des recueils de documents volumineux, mais aussi des textes très courts). Ces entrées se recoupent partiellement et leur nombre est supérieur à celui des textes ou recueils (à cause des différentes éditions d'une œuvre donnée), mais bien inférieur à celui des manuscrits (pour la plupart regroupés sous une seule entrée). La grande majorité des textes réunis par ces bibliographies exemplaires concerne la littérature profane et dans une moindre mesure la littérature religieuse ou les textes d'un savoir élaboré ou pratique ; les textes documentaires, en revanche, sont rares.

Il faudra sans doute supposer un ensemble de 2.000 à 3.000 textes littéraires profanes français écrits à travers quatre siècles entre $c a 1.100$ et $c a 1.500$. L'on peut également supposer que la quasi-totalité des textes littéraires conservés aujourd'hui a été répertoriée dans les bibliothèques du monde et que presque tous ont fait l'objet d'une édition, plus ou moins satisfaisante. Une évaluation chiffrée de la littérature religieuse ou des textes d'un savoir élaboré ou pratique est plus incertaine. Ces textes n'ont pas encore tous été identifiés et leur stade d'édition reste très partiel. Ces ensembles dépassent certainement la littérature profane, mais par un facteur sans doute inférieur à dix. La grande inconnue de la linguistique historique reste l'écrit documentaire dont le volume textuel est supérieur plusieurs milliers de fois à tous les autres genres confondus, mais dont le nombre de textes édités et analysés est très largement inférieur à celui des seuls textes littéraires. C'est donc là que le plus de découvertes reste à faire, même si ce domaine reste en dehors des préoccupations de la philologie littéraire.

L'étude des genres médiévaux doit prendre en considération, à tout moment, le modèle latin. Ce dernier est omniprésent pour les professionnels de l'écrit médiéval, presque tous bilingues et certainement conscients des différences autant sociolinguistiques qu'internes entre le code latin et le code français. Entre le début du XII e et le $\mathrm{XVI}^{\mathrm{e}}$ siècle, le français écrit a connu dans un processus constant une influence de plus en plus marquée du latin. Toutefois, le français garde son autonomie linguistique ; la syntaxe des chartes françaises, pour prendre un seul exemple, se détache nettement du modèle latin, même si la structure des actes reproduit les schémas textuels élaborés depuis l'Antiquité (cf. la synthèse de Buridant 2015 et le répertoire électronique en cours Duval/Vielliard s.d.; cf. également, pour l'aspect particulier, mais significatif des textes vernaculaires en caractères hébreux Kiwitt 2015).

Chaque genre entretient par ailleurs une relation particulière avec le phénomène des copies et des adaptations textuelles dans le processus de transmission. Les textes littéraires (profanes ou religieux) connaissent souvent de nombreuses copies indui- 
sant fréquemment des réinterprétations de contenu. Les textes d'un savoir élaboré répondent plus souvent au schéma de la compilation, intégrant différentes sources dans une nouvelle composition. Les textes documentaires enfin, sont rarement copiés plus de deux ou trois fois (copie contemporaine, transcription dans un cartulaire, copie du cartulaire), mais s’inscrivent par leur caractère sériel dans une suite ininterrompue de réadaptations d'un nombre limité de schémas textuels. Au-delà des attitudes divergentes, certaines constantes se relèvent pour les différents genres : en ligne générale, les copies transforment fortement les systèmes grapho-phonétique et morphologique de leurs antécédents, mais en respectent souvent les choix lexicaux ou syntaxiques.

Enfin, l'encodage matériel des textes varie fortement selon les époques; cela concerne les supports - parchemin, puis papier - et les outils d'écriture, l'évolution des écritures et des modèles de mise en page ou encore l'utilisation de cahiers ou de codex. Ces mêmes contraintes conditionnent la production des différents genres textuels, production qui est réalisée concrètement dans les mêmes établissements (à savoir les scriptoria ecclésiastiques et les chancelleries, puis au fur et à mesure, également dans des lieux de travail individuels). En revanche, les textes destinés à jouer un rôle de représentation connaissent l'emploi de matériaux plus précieux et sont écrits dans des écritures plus soignées, alors que les textes d'usage font toujours appel à des cursives moins soignées et à des supports moins coûteux. Cela s'observe également pour certains textes littéraires de genre mineur comme les fabliaux ou les farces.

\section{Les approches philologiques}

\subsection{L’édition}

Les textes français médiévaux dans toute leur diversité représentent un ensemble qui se prête aux études les plus diverses. Le point de départ obligé de toute analyse, linguistique ou littéraire, est la description des manuscrits et des textes ainsi que leur édition. L'ecdotique, la théorie de la critique textuelle, représente par conséquent le noyau de toute préoccupation philologique.

Les paramètres généraux sont simples et valent pour tous les genres : il s'agit de réunir l'intégralité des manuscrits qui peuvent être considérés comme des témoins d'un même texte, d'établir les liens de dépendance qui existent entre eux et de choisir un type d'édition donnée. Ces opérations ne sont pas faciles à mettre en œuvre puisqu'elles supposent une bonne connaissance des conditions de conservation du patrimoine écrit médiéval, une bonne compréhension de la langue d'oïl médiévale, une grande familiarité avec les traditions textuelles, des capacités de structuration et une grande rigueur dans le travail de détail. Mais au-delà de ces éléments liés à la formation et à la personnalité des chercheurs individuels, le choix du type d'édition soulève des problèmes théoriques et pratiques parfois insurmontables. 
La réflexion ecdotique a occupé de nombreux érudits depuis le XIX ${ }^{\mathrm{e}}$ siècle, travaillant essentiellement sur des textes littéraires (citons Karl Lachmann 1793-1851, Gaston Paris 1839-1903, Joseph Bédier 1864-1938, Mario Roques 1875-1961, Paul Maas 1880-1964, Félix Lecoy 1903-1997, Albert Henry 1910-2002, Gianfranco Contini 1912-1990, D’Arco Silvio Avalle 1920-2002 ou encore le regretté Cesare Segre 19282014, cf. Carapezza 2015). Grâce à la qualité et la précision de cette tradition interprétative, tous les cas de figure ont pu être envisagés et ont reçu une attention minutieuse.

Les idées de fond peuvent être grossièrement résumées ainsi :

(1) La première étape du recensement des manuscrits formant une tradition textuelle mène à l'établissement d'un stemma qui repose sur les dépendances respectives des manuscrits. Les méthodes pour établir un stemma ont pu être précisées depuis les travaux fondateurs de K. Lachmann (dès 1816) et de J. Bédier (Le Lai de l'Ombre 1913 et 1928, cf. Corbellari 1997, 505ss.). Des sondages effectués sur la structure textuelle, sur des passages et sur des lexèmes choisis peuvent donner des orientations précieuses pour éviter une comparaison intégrale des manuscrits. Mais même circonscrite, cette première étape reste indispensable et fastidieuse, puisqu'elle suppose une transcription au moins partielle des différents témoins. La stemmatologie contient par ailleurs un certain potentiel pour des interrogations d'ordre socio-historique. L'identification de la filiation et de la transmission des différents manuscrits permettrait de mieux cerner les lieux de leur genèse et d'établir des liens entre eux.

(2) Le choix pour établir une édition à partir de l'inventaire raisonné qu'est le stemma dépend fortement de la nature de la transmission textuelle :

- $\quad$ il peut s'avérer judicieux de choisir un manuscrit (relativement ancien et relativement complet) comme manuscrit de base et prendre en considération tous les autres manuscrits de la tradition seulement pour apporter des corrections ponctuelles ou pour témoigner d'une divergence lexicale ou textuelle (cf. Leonardi 2011) : c'est l'édition critique classique depuis les éditions de J. Bédier du Lai d'Ombre (1913) et, surtout, de la version anglonormande de la Chanson de Roland (1922) ;

- mais plus une tradition textuelle est éclatée, moins cette solution est satisfaisante. Il est alors possible de retenir plusieurs prototypes textuels en parallèle et de produire pour chacun d'entre eux une édition à part ;

- $\quad$ il est également possible de reconstituer un texte théorique à partir de différents manuscrits, cette solution - fréquente, notamment en Italie - se heurtant toutefois à la forte variation diatopique et diachronique présente dans chaque témoin ;

- enfin, il est possible d'éditer chacun des manuscrits parallèlement : c'est le principe de l'édition synoptique comme celle des huit manuscrits des Fabliaux (Noomen 1983-1998). Ce type d'édition est possible sous une forme diplomatique, c'est-à-dire la reproduction fidèle d'un maximum d'éléments du manuscrit, ou sous une forme critique, qui intervient dans la 
structuration du texte (ponctuation, paragraphes) et admet des émendations pour rendre le texte cohérent (tout en explicitant les diverses interventions).

Il est important de voir que la « qualité » d'une édition repose moins sur le choix du type d'édition - déterminé en grande partie par la nature de la tradition textuelle et par les finalités de l'édition (littéraire ou linguistique, grand public ou érudite) - que sur la cohérence de sa réalisation (choix des critères de transcription, prise en considération de la présentation matérielle des témoins, absence ou non de fautes de transcription, indication plus ou moins précise des interventions éditoriales, nombre et adéquation des émendations). Des paramètres objectifs pour l'évaluation d'une édition donnée sont difficiles à identifier ; notons que la tradition des études d'oîl ne dispose pas, à ce jour, d'un standard généralement reconnu (cf. l'orientation proposée par Bourgain/Guyotjeannin/Vielliard 2001-2002).

Par ailleurs, la révolution médiale de l'internet a ouvert de nouvelles perspectives éditoriales. La technologie informatique permet notamment la combinaison de différents types d'éditions dans un même environnement (édition diplomatique et édition critique combinées comme dans les Plus anciens documents linguistiques de la France [DocLing], édition diplomatique d'un témoin défini et édition critique de plusieurs prototypes textuels comme dans le projet de la Chanson d'Aspremont [Asprem]). Elle permet également d'intégrer la reproduction photographique des manuscrits (cf. Bozzi 2015) et elle ouvre des nouvelles voies pour la description linguistique des textes (cf. infra, 4.2).

L'informatique a pu renforcer ainsi la tendance actuelle qui consiste à prendre plus fortement en considération les manuscrits individuels comme témoins d'un état de langue et d'une interprétation précise d'un texte. Cette tendance a toujours existé dans la tradition italienne et s'est intensifiée par ailleurs suite à la discussion sur la «New Philology» (cf. Cerquiglini 1989; Glessgen/Lebsanft 1997). Pour l'analyse linguistique, les témoins individuels sont en effet plus immédiatement exploitables que les éditions critiques qui reposent sur différentes sources et opèrent des émendations plus ou moins explicites. La référence à un manuscrit unique est même indispensable pour toute étude grapho-phonétique ou morphologique. Toutefois, il reste important de pouvoir se référer à une édition critique lorsque l'on étudie le lexique ou la syntaxe d'un auteur donné car une critique textuelle soignée est indispensable à la bonne compréhension du texte. Les philologues linguistes tendent par conséquent à travailler sur des textes reposant sur peu de manuscrits, voire un seul (cf. l'exemple emblématique de Paul Meyer, Palumbo ms. ; cf. aussi Wilhelm 2015, §2).

Il est enfin important de souligner que l'édition n'est pas séparable de sa description linguistique puisque celle-ci intervient obligatoirement dans l'établissement du texte. Ajoutons que les exploitations souhaitées d'une édition par son auteur conditionnent la nature de celle-ci : la philologie linguistique préfère les éditions de manuscrits individuels tandis que la philologie littéraire préfère les éditions critiques voire reconstructives permettant une lecture suivie. Une édition n'est donc jamais 
indépendante de son utilisation (parfois seulement potentielle), et elle n'est pas, et ne devrait jamais être, une fin en soi (cf. Beltrami 2013, A che serve un'edizione critica ?) : la philologie reste stérile si elle n'interprète pas les textes, soit dans une optique littéraire, linguistique, ou historique.

\subsection{La description linguistique}

Les éditions médiévistes sont traditionnellement accompagnées d'une introduction comportant des éléments descriptifs concernant autant la tradition textuelle et l'ancrage littéraire que les aspects grapho-phonétiques et morphologiques significatifs du texte. L'établissement du texte est enfin suivi d'un glossaire d'ampleur et d'exigence méthodologique très variable. Ces éléments sont généralement considérés comme appartenant au travail philologique. Cela s'explique par le fait, nous l'avons dit, que l'édition suppose une analyse linguistique préparatoire, pour cerner la valeur des graphèmes, appréhender les variations flexionnelles, comprendre chaque unité lexicale (non seulement pour s'assurer de la cohérence sémantique du texte mais aussi pour segmenter correctement les mots, structurer le contenu par la ponctuation et opérer, si nécessaire, des émendations). Par ailleurs, l'analyse linguistique fournit des informations essentielles pour la localisation et, dans une certaine mesure aussi, pour la datation autant du texte originel (et perdu) que celle des copies transmises. Ce travail préparatoire n'est pas toujours apparent, mais il peut aussi mener, dans une édition donnée, à la rédaction de chapitres linguistiques volumineux.

De manière plus générale, soulignons que tout travail linguistique reposant directement sur des sources textuelles médiévales peut être considéré comme 'philologique'. En effet, la prise en considération de la critique textuelle est indispensable à l'analyse linguistique des témoins médiévaux, où chaque paramètre essentiel - tels le lieu, la date, l'auteur ou le genre textuel - contient une dimension interprétative, souvent délicate. La philologie se réalise également dans une analyse linguistique, même thématique, basée sur un corpus textuel, même si ce corpus a été établi par un tiers. En fin de compte, l'étude de la langue médiévale est tout aussi inséparable d'un socle philologique que l'édition d'un texte ne l'est de son analyse linguistique.

\subsubsection{La question de l'optique différentielle}

Selon les domaines du langage concernés, l'analyse linguistique dans un cadre éditorial poursuit des finalités variables et appelle des angles d'approches différents. Mais dans tous les cas il faut distinguer les particularités d'un texte ou d'un manuscrit et les caractéristiques de la langue à un moment donné, dans une région ou dans un genre définis. La description philologique s’inscrit donc par définition dans une logique différentielle : qu'est-ce qui est propre au texte/manuscrit en question ? Cette 
interrogation suppose l'existence d'une référence qui puisse servir de point de comparaison. Or, en l'absence d'un standard médiéval, ce terme de comparaison devrait être l'ensemble des paramètres propres au diasystème du français à l'époque concernée, dans toute sa variation. Concrètement, il faudrait mesurer un texte en le comparant à tous les autres textes oïliques analysés, autrement dit à l'état général de nos connaissances sur la langue médiévale. Toutefois, si l'on dispose aujourd'hui d'excellents dictionnaires qui constituent une référence solide pour le lexique, nulle synthèse n'existe pour les domaines grapho-phonétique, morphologique et syntaxique. Tout éditeur de texte se retrouve donc en face de plusieurs milliers d'autres éditions de texte, contenant chacune un certain nombre d'informations linguistiques - sans compter les données non exploitées par l'éditeur - et doit se positionner dans sa propre description par rapport à cette mosaïque insaisissable. En conséquence, la grande majorité des descriptions linguistiques accompagnant les éditions renoncent à l'exigence de contrastivité et optent pour une procédure traditionnelle et stéréotypée, en s’alignant sur quelques modèles antérieurs considérés comme réussis.

Il est difficile de remédier à cet état de fait puisque les problèmes évoqués sont de nature structurelle. Mais il est important d'en prendre conscience pour pouvoir opérer des choix individuels cohérents et pour pouvoir développer dans les années à venir une stratégie de recherche qui pourrait mener à la fois à une meilleure connaissance de la langue médiévale et à un meilleur fondement du travail différentiel sur les textes et manuscrits.

\subsubsection{L’étude grapho-phonétique}

Il est regrettable de constater que, traditionnellement, la description grapho-phonétique des introductions aux éditions de texte ne se place pas dans une optique phonologique. Celle-ci, pourtant déjà ancienne, permettrait de cerner le statut des graphèmes et de leur valeur dans une logique de système. Il est certain que les scriptae médiévales produisent des formes graphématiques qui n'ont parfois aucune correspondance naturelle avec une prononciation donnée et qui sont le reflet d'interférences entre différentes formes écrites, par exemple :

- $\quad$ la forme a(i)nrme 'âme', par interférence entre an(e)me et arme (cf. Pfister 1993, 37),

- dans la scripta lorraine, il existe les variantes estaule (avec vocalisation régionale de /b/ préconsonantique), estauvle (avec un élément svarabhaktique peut-être lié à l'oral), estable (forme latinisante ou/et influencée par des régions voisines sans vocalisation de la bilabiale) et, enfin, estauble (emprunt de la scripta bourguignonne où la forme reflète une interférence entre les types écrits <able> et <aule>, cf. Glessgen 2008, 450).

Néanmoins le cadrage phonologique permettrait de dépasser le caractère anecdotique de remarques graphocentriques et d'approcher la variation graphématique de manière plus rigoureuse. 
Les ouvrages qui peuvent orienter l'analyse grapho-phonétique sont peu nombreux : l'analyse d'ordre phonologique de J.-M. Pierret (1994), la description phonétique de La Chaussée (1989), l'ouvrage fondateur de Fouché (1952-1969) ainsi que les deux Atlas de Dees $(1980$; 1987) qui représentent, malgré leurs défauts méthodologiques, les seuls répertoires généraux de la variation dans les scriptae oïliques. Le plus important desideratum de la recherche serait de réunir - idéalement sur une base de données, par conséquent évolutive - les éléments descriptifs concernant la graphophonétique médiévale éparpillés dans les milliers d'éditions existantes. Une telle synthèse demanderait un effort considérable mais rendrait enfin sérieusement envisageable un travail différentiel.

Enfin, il est important de prendre conscience du fait que la variation diatopique, omniprésente dans les scriptae médiévales, répond en même temps à des critères sociologiques. Dans un même espace géographique, l'on peut trouver des scriptae plus ou moins régionalisées, selon le prestige des rédacteurs, selon la «portée communicative» des textes (Völker 2003) ou selon les genres textuels. Un «lieu d'écriture » médiéval n’est donc pas simplement déterminé par un lieu géographique et il peut même être délocalisé, comme c'est le cas des grandes chancelleries princières (Glessgen 2008 ; Videsott 2013). Soulignons encore que toute étude scriptologique contient une dimension interprétative ecdotique, notamment dans le cas de transmissions textuelles complexes (p. ex. Zinelli 2008).

\subsubsection{L'étude morphologique et syntaxique}

Le cas de la morphologie flexionnelle est très semblable à celui de la grapho-phonétique. Il existe un important relevé de près de 20.000 graphies verbales (et 500.000 occurrences) du français médiéval, établi par Robert Martin (cf. Martin/Kunstmann 2004). Mais ce relevé est actuellement en cours d'élaboration et ne comporte que partiellement des informations diasystématiques.

Pour les marques grammaticales et la syntaxe, en revanche, la configuration est radicalement différente : les fonctions morphologiques et la structure de la phrase ne font pas partie, traditionnellement, des éléments descriptifs des éditions. L'essor des études syntaxiques date des années 1960, à un moment où les modèles éditoriaux étaient déjà établis depuis longtemps. Curieusement, cette absence ne porte pas un fort préjudice à l'établissement des textes, puisque la cohésion du système grammatical de la langue fait que sa variation ne s'inscrit que très peu dans les dimensions diatopique ou diastratique. Elle suit, au moins pour la syntaxe complexe, les contraintes et les nécessités expressives des genres textuels et des traditions de discours.

Il est néanmoins à déplorer que la philologie linguistique ait largement sousexploité jusqu'aujourd'hui l'analyse syntaxique des genres textuels. Le manque d'intérêt flagrant pour l'analyse grammaticale de l'ancienne langue se reflète même dans la diffusion des livres. On pense notamment à la faible disponibilité d'ouvrages de 
référence d'orientation moderne (c'est le cas de Systèmes morphologiques de l'ancien français d'Andrieux et Baumgartner 1983 ou encore de la Grammaire nouvelle de l'ancien français de C. Buridant 2000, ouvrages tous deux épuisés) au profit de manuels scientifiquement dépassés et conçus pour la préparation des concours de l'enseignement secondaire en France. La recherche sur la syntaxe du français médiéval a certes connu un développement réel dans les dernières décennies. Mais autant dans le domaine de la morphologie que de la syntaxe, les deux traditions philologique et linguistique se sont éloignées de manière particulièrement flagrante.

\subsubsection{L'étude lexicale}

La lexicologie constitue la part linguistique sans doute la plus présente dans la philologie éditoriale. Pourtant, c'est ici que les points de vue linguistiques et philologiques divergent le plus. La lexicologie médiéviste suppose la compréhension de chaque lexème dans un texte ou un manuscrit donné, et la vérification de sa distribution dans le temps et dans l'espace pour établir un glossaire. Seule la lexicographie de référence garantit dans cette optique une bonne compréhension des textes et peut déterminer de manière non subjective la nomenclature du glossaire. Elle permet également l'établissement de définitions (et non d'équivalences en français moderne) qui s'inscrivent dans l'excellente tradition lexicologique monolingue du français. Cela concerne notamment l'utilisation du FEW, à présent disponible en ligne (<www.atilf. fr/few>), à côté des dictionnaires historiques (Gdf, TL, DEAF, DMF, AND).

Or, les conseils d'établissement de glossaires formulés par des lexicologues (Buridant 1999 ; Chambon 2006) n'ont malheureusement pas eu l'impact souhaitable sur la pratique éditoriale du français médiéval (cf. plus récemment Roques 2011 ; Buridant s. p. ; Möhren 2015). Il n'y a en effet quasiment que les auteurs rattachés directement ou indirectement, par leurs maîtres, aux centres actuels de lexicographie qui produisent des glossaires textuels équilibrés et cohérents. La situation est encore plus grave pour l'analyse des matériaux toponymiques et anthroponymiques présents dans les textes (cf. toutefois les nombreuses études exemplaires de J.-P. Chambon, p. ex. 2014).

Ce constat quelque peu déroutant est récemment contrebalancé par l'essor de la lexicographie informatique. Le Dictionnaire Électronique de Chrétien de Troyes (DÉCT), de P. Kunstmann (2013), place la description du vocabulaire de cet auteur emblématique dans un cadre lexicographique moderne et prépare le terrain à des analyses de nature interprétative. Par ailleurs, le vocabulaire contenu dans le corpus des Plus anciens documents linguistiques de la France (DocLing) est à présent intégré dans la base lexicographique du DEAFPré et peut être interrogé à partir de celle-ci. On espère que se tisseront ainsi de nouveaux liens entre les textes et les outils de référence et qu'ils permettront une description lexicologique plus adéquate des textes. 
Notons enfin qu'il existe, pour le français médiéval, une grande tradition d'études lexicologiques portant sur des auteurs définis ou des thématiques spécifiques (telles que les termes liés à la négation, les formules de salutation, le vocabulaire agricole ou des sentiments) et qui se réclament à juste titre de la philologie.

\subsubsection{Problèmes interprétatifs}

L'établissement des textes et la description de leurs particularités linguistiques se placent naturellement dans un contexte d'interrogations plus générales. Ainsi, la relation entre l'oral et l'écrit contient un réel potentiel de recherche pour l'analyse des textes médiévaux. Même si l'oral n'est pas immédiatement saisissable, il est omniprésent dans les textes écrits qui s'en détachent de manière plus ou moins marquée. Il serait donc possible de déceler dans les différents genres textuels les deux tendances d'un encodage reflétant les structures de l'oral et celle d'une complexification textuelle de conception scripturale.

Cette opposition rejoint la variation diasystématique dans les scriptae. La régionalité de l'écrit médiéval reste également un domaine d'étude particulièrement porteur, même s'il connaît une tradition déjà ancienne. Les récentes possibilités de quantification permettent en effet d'établir avec une réelle précision le degré de régionalité ou de dialectalité d'un texte ou manuscrit donné, ce qui permet de le replacer avec justesse dans le contexte communicatif de l'époque.

Enfin, les interrogations sur le rôle des acteurs individuels dans le paysage de l'écrit permettraient non seulement de mettre en relief l'individualité langagière des textes ou manuscrits singuliers, mais aussi de mieux cerner l'usage et le changement linguistiques. L'identification de la part d'idiosyncrasie des scribes et des auteurs jetterait ainsi un pont entre philologie linguistique et littéraire.

\section{Les lieux de la philologie linguistique}

Il faut enfin prendre en considération la question des "lieux » où s'exprime la philologie linguistique. Elle emprunte les vecteurs essentiels des sciences modernes tels que l'enseignement, les publications, les rencontres scientifiques ou encore les associations savantes.

Concrètement, les grands dictionnaires de l'ancienne langue restent un des lieux les plus actifs de la philologie linguistique. Par ailleurs, les travaux spécifiques connaissent une bonne présence parmi les publications monographiques, les articles et comptes rendus de revues ainsi que parmi les réalisations en philologie électronique (cf. pour le genre particulièrement développé du compte rendu, Roques 2015).

En revanche, si les rencontres scientifiques sont légion en philologie purement éditoriale ou littéraire, elles sont bien moins fréquentes en philologie linguistique (cf. 
pourtant sa présence constante dans les congrès triennaux de la Société de Linguistique Romane). Ce constat est corrélé avec la prédominance littéraire dans les associations philologiques (comme la Société des anciens textes français, la Société de langue et de littérature médiévales d'oc et d'oül ou la Société internationale renardienne). Dans l'enseignement, enfin, la philologie linguistique reste périphérique et il n'existe que très peu de manuels qui s'inscrivent dans son optique (p. ex. Duval 2009; Glessgen 2012a, 420-456).

Le constat général est donc double : une réelle existence de la discipline dans les vecteurs de la recherche de pointe s'oppose à une assez faible présence dans l'enseignement et dans les cercles habituels de communication scientifique. L'on peut souhaiter que s'opère un rééquilibrage dont la réalisation supposerait toutefois que la linguistique théorique autant que la philologie littéraire reconnaissent à la philologie linguistique sa légitimité de droit.

\section{Bibliographie}

ALF = Gilliéron, Jules/Edmont, Edmond (1902-1910), Atlas linguistique de la France, 15 vol., Paris, Champion.

AND = Stone, Louise/Rothwell, William (edd.) (1977-1992), Anglo-Norman Dictionary, London, Modern Humanities Research Association ; Rothwell, William/Gregory, Stewart/Trotter, David (edd.) (2005), Anglo-Norman Dictionary. Second edition, ib. [A-E] ; Trotter, David (ed.) (2009-), AngloNorman Dictionary (version en ligne) [F-] <www.anglo-norman.net> (30.04.2015).

Andrieux, Nelly/Baumgartner, Emmanuelle (1983), Systèmes morphologiques de l'ancien français. A. Le verbe, Bordeaux, Éd. Bière.

Asprem = La chanson d'Aspremont. Projet d'édition électronique <chansondaspremont.eu> (30.04.2015).

Baum, Richard (2003), Periodisierung in der romanistischen Sprachgeschichtsschreibung, in : Gerhard Ernst et al. (edd.), Romanische Sprachgeschichte / Histoire linguistique de la Romania, vol. 1, Berlin/New York, de Gruyter, 45-53.

Beltrami, Pietro (2013), A che serve un'edizione critica ? Leggere i testi della letteratura romanza medievale, Bologna, il Mulino.

Berschin, Helmut/Berschin, Walter/Schmidt, Rolf (1981), «Augsburger Passionslied ». Ein neuer romanischer Text des $X$. Jahrhunderts, in : Walter Berschin/Reinhard Düchting (edd.), Lateinische Dichtungen des X. und XI. Jahrhunderts. Festgabe für Walther Bulst zum 80. Geburtstag, Heidelberg, Schneider, 251-279.

Bossuat, Robert (1951), Manuel bibliographique de la littérature française du Moyen Âge, Melun, Librairie d’Argences [Bossuat, Robert (1955), Supplément [1949-1953] ; Bossuat, Robert/ Monfrin, Jacques (1961), Second supplément [1954-1960] ; Vielliard, Françoise/Monfrin, Jacques (1986), Troisième supplément [1960-1980].

Bourgain, Pascale/Guyotjeannin, Olivier/Vielliard, Françoise (2001-2002), Conseils pour l'édition des textes médiévaux, 3 vol., Paris, Comité des travaux historiques et scientifiques/ENC.

Bozzi, Andrea (2015), Entre texte et image : la méthode de Pise, in : David Trotter (ed.), Manuel de la philologie de l'édition, Berlin/Boston, de Gruyter.

Buridant, Claude (1999), Proposition de protocole pour la confection de lexiques de français préclassique, Le Français préclassique 6, 115-133. 
Buridant, Claude (2000), Grammaire nouvelle de l'ancien français, Paris, SEDES.

Buridant, Claude (2015), Édition et traduction, in : David Trotter (ed.), Manuel de la philologie de l'édition, Berlin/Boston, de Gruyter.

Buridant, Claude (s. p.), Éditions de textes médiévaux en français et lexicographie : variantes, glossairistique et traduction, in : $27^{\mathrm{e}}$ CILPR, Nancy, section17.

Capusso, Maria Grazia (2007), La produzione franco-italiana dei secoli XIII e XIV : convergenze letterarie e linguistiche, in : Renato Oniga/Sergio Vatteroni (edd.), Plurilinguismo letterario, Soveria Mannelli, Rubbettino, 159-204.

Carapezza, Francesco (2015), Entre théorie et pratique en ecdotique galloromane, in : David Trotter (ed.), Manuel de la philologie de l'édition, Berlin/Boston, de Gruyter.

Carles, Hélène (2011), L'émergence de l'occitan pré-textuel. Analyse linguistique d'un corpus auvergnat (IXe-XIe siècles), Strasbourg, SLiR/ÉLiPhi.

Cerquiglini, Bernard (1989), Éloge de la variante. Histoire critique de la philologie, Paris, Seuil.

Chambon, Jean-Pierre (1998), L'identité langagière des élites cultivées d'Arvernie autour de l'an Mil et la "scripta latina rustica » : réflexions à propos du «breve de libros » du chapitre cathédral de Clermont (984-1110), Revue de Linguistique Romane 62, 381-408.

Chambon, Jean-Pierre (2006), Lexicologie et philologi : réflexion sur les glossaires d'éditions de textes (français médiéval et préclassique, ancien occitan), Revue de Linguistique Romane 70, 123-142.

Chambon, Jean-Pierre (2014), Contributions à la toponymie de la Lozère, principalement d'après les sources médiévales, Revue de Linguistique Romane 78, 147-202.

Chambon, Jean-Pierre/Greub, Yan (2000), Données nouvelles pour la linguistique gallo-romane: les légendes monétaires mérovingiennes, Bulletin de la Société de Linguistique de Paris 95 , 147-182.

Chambon, Jean-Pierre/Müller, Wulf (2013), Le nom de lieu déhydronomique «Lutran » (Haut-Rhin) : un indice du francoprovençal submergé dans le Sundgau alsacien, in : Albrecht Greule et al. (edd.), La région de Bâle et les rives du Rhin de l'Antiquité au Moyen Âge, Stuttgart, Kohlhammer, 107117.

Contini, Gianfranco (2007), Frammenti di filologia romanza. Scritti di ecdoctica e linguistica (19321989), ed. Giancarlo Breschi, 2 vol., Firenze, Edizioni del Galluzzo.

Corbellari, Alain (1997), Joseph Bédier, écrivain et philologue, Genève, Droz.

DEAF = Baldinger, Kurt/Möhren, Frankwalt/Städtler, Thomas (edd.) (1979-), Dictionnaire étymologique de l'ancien français, Tübingen, Niemeyer/Berlin et al., de Gruyter.

DEAFPré = Dictionnaire étymologique de l'ancien français. Version en ligne <http://deaf-server.adw. uni-heidelberg.de>.

Dees, Antonij (1980), Atlas des formes et des constructions des chartes françaises du $13^{e}$ siècle, Tübingen, Niemeyer.

Dees, Antonij (1987), Atlas des formes linguistiques des textes littéraires de l'ancien français, Tübingen, Niemeyer.

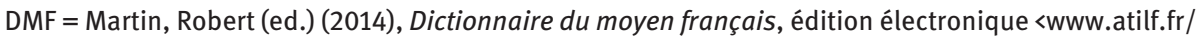
dmf> (30.04.2015).

DocLing = Les plus anciens documents linguistiques de la France (DocLing). Édition électronique, dirigée par Martin Glessgen, en partenariat avec Frédéric Duval et Paul Videsott. Deuxième édition entièrement revue et élargie ( $\left.{ }^{2} 2013\right)<$ rose.uzh.ch/docling> (30.04.2015).

Duval, Frédéric (ed.) (2006), Pratiques philologiques en Europe. Actes de la journée d'étude à l'École des chartes le 23 septembre 2003, Paris, École des chartes.

Duval, Frédéric (2009), Le français médiéval, Turnhout, Brepols.

Duval, Frédéric (2015), Les éditions de textes du XVIIe siècle, in : David Trotter (ed.), Manuel de la philologie de l'édition, Berlin/Boston, de Gruyter. 
Duval, Frédéric/Vielliard, Françoise (s. d.), Le miroir des classiques (Éditions en ligne de l'Ecole des chartes, 17), http://elec.enc.sorbonne.fr/miroir (30.04.2015).

Fouché, Pierre (1952-1969), Phonétique historique du français, 3 vol., Paris, Klincksieck.

$\mathrm{Gdf}=$ Godefroy, Frédéric (1880-1902), Dictionnaire de l'ancienne langue française et de tous ses dialectes du ix $x^{e}$ au xv siècle, 10 vol., Paris, Bouillon.

Glessgen, Martin (2008), Les lieux d'écriture dans les chartes lorraines du XIII siècle, Revue de Linguistique Romane 75, 391-468.

Glessgen, Martin (2012a), Linguistique romane. Domaines et méthodes en linguistique française et romane, $2^{\mathrm{e}}$ éd. intégralement remaniée $\left({ }^{1} 2007\right)$, Paris, Colin.

Glessgen, Martin (2012b), Trajectoires et perspectives en scriptologie galloromane, Medioevo Romanzo 36, 5-23.

Glessgen, Martin/Lebsanft, Franz (edd.) (1997), Alte und neue Philologie, Tübingen, Niemeyer.

Goebl, Hans (2012), L'aménagement scripturaire du domaine d'oïl médiéval à la lumière des calculs de localisation d'Anthonij Dees effectués en 1983 : une étude d'inspiration scriptométrique, publication électronique (<medioevoromanzo.it>, seminario, 2011, Il problema della scripta) (30.04.2015).

GRLMA = Jauss, Hans Robert, et al. (edd.) (1968-), Grundriß der romanischen Literaturen des Mittelalters, Heidelberg, Winter.

Grübl, Klaus (2013), La standardisation du français au Moyen Âge : point de vue scriptologique, Revue de Linguistique Romane 77, 343-383.

Hilty, Gerold (1994), La "Passion d'Augsbourg », reflet d'un poème occitan du Xe siècle, in : Jacqueline Cerquiglini-Toulet/Olivier Collet (edd.), Mélanges de philologie et de littérature médiévales offerts à Michel Burger, Genève, Droz, 231-244.

Holtus, Günter/Wunderli, Peter (2005), Franco-italien et épopée franco-italienne, in : Hans Robert Jauss et al. (edd.), Grundriß der romanischen Literaturen des Mittelalters, vol. 3, t. 1/2, fasc. 10, Heidelberg, Winter.

InvSyst = Frank, Barbara/Hartmann, Jörg (1997), Inventaire systématique des premiers documents des langues romanes, 5 vol., Tübingen, Narr.

Kiwitt, Marc (2015), L'ancien français en caractères hébreux, in : David Trotter (ed.), Manuel de la philologie de l'édition, Berlin/Boston, de Gruyter.

Klapp, Otto/Klapp-Lehmann, Astrid (1960-), Bibliographie der französischen Literaturwissenschaft, Frankfurt am Main, Klostermann.

Kristol, Andres (2002), Traces toponymiques du francoprovençal submergé en Suisse alémanique occidentale, Vox Romanica 61, 222-244.

Kunstmann, Pierre (2013), Dictionnaire Électronique de Chrétien de Troyes, LFA/Université d’Ottawa, ATILF/Université de Lorraine, www.atilf.fr/dect (30.04.2015).

La Chaussée, François (de) (1989), Initiation à la phonétique historique de l'ancien français, Paris, Klincksieck.

Leonardi, Lino (2011), Il testo come ipotesi (critica del manoscritto-base), Medioevo Romanzo 35, 5-34.

Martin, Robert/Kunstmann, Pierre (2004), Base de graphies verbales <www. atilf.fr/bgv> (actuellement indisponible).

Minervini, Laura (2010), Le français dans l'Orient Latin (XIII -XIVe siècles). Éléments pour la caractérisation d'une « scripta » du Levant, Revue de Linguistique Romane 74, 121-198.

Minervini, Laura (2012), Les emprunts arabes et grecs dans le lexique français d'Orient (XIIIe-XIVe siècles), Revue de Linguistique Romane 76, 99-197.

Möhren, Frankwalt (2015), L'art du glossaire d'édition, in : David Trotter (ed.), Manuel de la philologie de l'édition, Berlin/Boston, de Gruyter.

Noomen, Willem (1983-1998), Nouveau recueil complet des fabliaux (NRCF), 10 vol., Assen, Van Gorcum. 
Palumbo, Giovanni (ms.), Sur les pas de Paul Meyer : l'édition des textes médiévaux entre théorie et pratique.

Pfister, Max (1993), Scripta et koinè en ancien français aux XII et XIII siècles, in : Pierre Knecht/ Zygmunt Marzys (edd.), Écriture, langues communes et normes (Neuchâtel, 21-23 septembre 1988), Genève, Droz/Neuchâtel, Faculté des Lettres, 17-41 [réimprimé in : Glessgen, Martin/ Schweickard, Wolfgang (edd.), Ex traditione innovatio. Miscellanea in honorem Max Pfister septuagenarii oblata, vol. 1, Darmstadt, Wissenschaftliche Buchgesellschaft, 2002, 99-123].

Pierret, Jean-Marie (1994), Phonétique historique du français et notions de phonétique générale, Louvain-la-Neuve, Peeters.

Pignon, Jacques (1960), L'évolution phonétique des parlers du Poitou (Vienne et Deux-Sèvres), 2 vol., Paris, d'Artrey.

Rézeau, Pierre (2013), Les Noëls en France aux XVe et XVI siècles. Édition et analyse, Strasbourg, SLiR/ ÉLiPhi.

Roques, Gilles (2011), Typologie des glossaires des éditions de textes de français médiéval, in : Eurolab, Dynamique des langues vernaculaires dans l'Europe de la Renaissance. Acteurs et lieux, Séminaire doctoral $n^{\circ} 1$ : Langues et glossaires (Liège, 17 mai 2010, <eurolab.meshs.fr〉) (30.04.2015).

Roques, Gilles (2015), Défense et illustration du compte rendu scientifique, in : David Trotter (ed.), Manuel de la philologie de l'édition, Berlin/Boston, de Gruyter.

RSG = Gerhard Ernst et al. (edd.), Romanische Sprachgeschichte / Histoire linguistique de la Romania. Ein internationales Handbuch zur Geschichte der romanischen Sprachen und ihrer Erforschung I Manuel internationel d'histoire linguistique de la Romania (Handbücher zur Sprach- und Kommunikationswissenschaft, HSK 23/1-23/3), Berlin/New York, Mouton de Gruyter, 3 vol. (2003, 2006, 2008).

Stotz, Peter (1996-2004), Handbuch zur lateinischen Sprache des Mittelalters, 5 vol., München, Beck.

$\mathrm{TL}=$ Tobler, Adolf/Lommatzsch, Erhard (poursuivi par Hans H. Christmann et Richard Baum) (19251936 ; 1954-2002), Altfranzösisches Wörterbuch, 11 vol., Berlin, Weidmann/Wiesbaden, Steiner.

Trotter, David (ed.) (2012), Present and future research in Anglo-Norman. Proceedings of the Aberystwyth Colloquium, July 2011, Aberystwyth, Anglo-Norman Online Hub.

Trotter, David (2013 [2014]), « Deinz certeins boundes ». Where does Anglo-Norman begin and end?, Romance Philology 67, 139-177 ; version en ligne : http://brepols.metapress.com/content/ m0814673846344t3/fulltext.pdf (30.04.2015).

Trotter, David (2015), Coup d'œil sur les scriptae médiévales et les textes qui les représentent, in : Maria Iliescu/Eugeen Roegiest (edd.), Manuel des anthologies, corpus et textes romans, Berlin/ Boston, de Gruyter.

TypSources = Génicot, Léopold (ed.) (1972-), Typologie des sources du Moyen Âge occidental, Louvain/Turnhout, Brepols.

Vachon, Claire (2010), Le changement linguistique au XVI siècle. Une étude basée sur des textes littéraires français, Strasbourg, SLiR/ÉLiPhi.

Videsott, Paul (2013), Les débuts du français à la Chancellerie royale : analyse scriptologique des chartes de Philippe III (1270-1285), Revue de Linguistique Romane 77, 3-50.

Völker, Harald (2003), Skripta und Variation. Untersuchungen zur Negation und zur Substantivflexion in altfranzösischen Urkunden der Grafschaft Luxemburg (1237-1281), Tübingen, Niemeyer.

Wilhelm, Raymund (2015), L'édition de texte - entreprise à la fois linguistique et littéraire, in : David Trotter (ed.), Manuel de la philologie de l'édition, Berlin/Boston, de Gruyter.

Zinelli, Fabio (2008), Tradizione " mediterranea » e tradizione italiana del "Livre dou Tresor », in : Irene M. Scariati (ed.), A scuola con Ser Brunetto, Firenze, Galluzzo, 35-89.

Zufferey, François (2006), Robert de Boron et la limite nord du francoprovençal, Revue de Linguistique Romane 70, 431-469. 



\section{Table des matières}

Claudia Polzin-Haumann et Wolfgang Schweickard

0 Introduction -1

\section{Le français dans l'histoire}

Philipp Burdy

1 Le français dans l'histoire : depuis ses origines jusqu'au XVIe siècle -11

Christian Schmitt

2 Le français dans l'histoire : du XVII'e siècle à nos jours — 39

Gerhard Ernst

3 La diachronie dans la linguistique variationnelle du français -72

Hélène Carles et Martin Glessgen

4 La philologie linguistique et éditoriale -108

\section{Le français moderne}

Eva Martha Eckkrammer et Stéphanie Lescure

5 Aménagement linguistique et défense institutionnalisée de la langue :

France -133

Dietmar Osthus

6 Linguistique populaire et chroniques de langage : France -160

Ursula Reutner

7 Aménagement linguistique et défense institutionnalisée de la langue :

Francophonie - 171

Carolin Patzelt

8 Linguistique populaire et chroniques de langage : Francophonie 196

Felix Tacke

9 Aménagement linguistique et défense institutionnalisée de la langue :

les français régionaux et les langues des minorités — 216 
Judith Visser

10 Linguistique populaire et chroniques de langage : les français régionaux et les langues des minorités — 242

Thomas Krefeld

11 L'immédiat, la proximité et la distance communicative -262

Anja Overbeck

12 La communication dans les médias électroniques - 275

Kristina Bedijs

13 Langue et générations : le langage des jeunes — 293

Annette Gerstenberg

14 Langue et générations : enjeux linguistiques du vieillissement — 314

Elmar Schafroth

15 Sexe et genre -334

Joachim Lengert

16 Les français régionaux — 365

Esme Winter-Froemel

17 Le français en contact avec d'autres langues - 401

Elmar Eggert

18 Le français dans la communication scientifique et internationale -432

Johannes Kramer et Aline Willems

19 Le français dans le monde : Europe -457

Edith Szlezák

20 Le français dans le monde : Canada -478

Sabine Diao-Klaeger

21 Le français dans le monde : Afrique -505

Pierre Swiggers

22 Grammaticographie $-\mathbf{5 2 5}$

Xavier Gouvert et Ulrike Heidemeier

23 Lexicographie -556 


\section{Tendances méthodologiques et didactiques actuelles}

Paul Gévaudan

24 La linguistique cognitive -585

Britta Thörle

25 La linguistique appliquée - 618

Christiane Fäcke

26 Le français dans l'enseignement scolaire et universitaire - 639

Christina Reissner

27 La recherche en plurilinguisme -659

Achim Stein

28 Linguistique française et ressources électroniques - 681

Michael Schreiber

29 Traduction -696

Index -717 